\title{
Anterior Cingulate Glutamate Levels Related to Clinical Status Following Treatment in First-Episode Schizophrenia
}

\author{
Alice Egerton*,', Stefan Brugger ${ }^{1,2}$, Marie Raffin', Gareth J Barker ${ }^{3}$, David J Lythgoe ${ }^{3}$, Philip K McGuire ${ }^{1,5}$ \\ and James $M$ Stone ${ }^{1,4,5}$ \\ 'Department of Psychosis Studies, Institute of Psychiatry, King's College London, De Crespigny Park, London, UK; '2St George's Hospital, University \\ of London, London, UK; ${ }^{3}$ Department of Neuroimaging, Centre for Neuroimaging Sciences, Institute of Psychiatry, King's College London, \\ De Crespigny Park, London, UK; ${ }^{4}$ Experimental Medicine, Imperial College London, Hammersmith Hospital, London, UK
}

\begin{abstract}
Many patients with schizophrenia show a limited symptomatic response to treatment with dopaminergic antipsychotics. This may reflect the additional involvement of non-dopaminergic neurochemical dysfunction in the pathophysiology of the disorder. We tested the hypothesis that brain glutamate levels would differ between patients with first-episode psychosis who were symptomatic compared with those with minimal symptoms following antipsychotic treatment. Proton magnetic resonance spectroscopy (IH-MRS) spectra were acquired at 3 Tesla in the anterior cingulate cortex and left thalamus in 15 patients with first-episode psychosis in symptomatic remission, and 17 patients with first-episode psychosis who were still symptomatic following at least one course of antipsychotic treatment. Metabolite levels were estimated in ratio to creatine $(\mathrm{Cr})$ using LCModel. Levels of glutamate/ $\mathrm{Cr}$ in the anterior cingulate cortex were significantly higher in patients who were still symptomatic than in those in remission $(T(30)=3.02 ; P=0.005)$. Across the entire sample, higher levels of glutamate/ $\mathrm{Cr}$ in the anterior cingulate cortex were associated with a greater severity of negative symptoms $(r=0.42$; $P=0.017)$ and a lower level of global functioning $(r=-0.47 ; P=0.007)$. These findings suggest that clinical status following antipsychotic treatment in schizophrenia is linked to glutamate dysfunction. Treatment with compounds acting on the glutamatergic system might therefore be beneficial in patients who respond poorly to dopaminergic antipsychotics.

Neuropsychopharmacology (2012) 37, 25I5-2521; doi:I0.1038/npp.2012.1 I3; published online 4 July 2012
\end{abstract}

Keywords: psychosis; magnetic resonance spectroscopy; glutamate; treatment response; anterior cingulate cortex; thalamus

\section{INTRODUCTION}

In the treatment of schizophrenia with antipsychotic drugs, on average around $60 \%$ occupancy of brain D2 dopamine receptors is required to produce a therapeutic response (Kapur et al, 2000). However, a substantial proportion of patients still show a poor response even when D2 occupancy is at this level (Pilowsky et al, 1993). This may reflect the importance of non-dopaminergic neurochemical dysfunction in the pathophysiology of schizophrenia. Increasing evidence from pharmacological, neuropharmacological, and genetic studies suggests that the primary deficit in schizophrenia may be a developmentally driven abnormality in the glutamate system (see (Egerton et al, 2012; Stone et al, 2007)). Several clinical trials have demonstrated the efficacy

\footnotetext{
*Correspondence: Dr A Egerton, Department of Psychosis Studies, Institute of Psychiatry, King's College London, De Crespigny Park, London SE5 8AF, UK. Tel: + I 442078480879 , Fax: + I 44207848 0976, E-mail: Alice.Egerton@kcl.ac.uk

${ }^{5}$ These authors contributed equally to this work

Received I6 April 2012; revised II May 2012; accepted 30 May 2012
}

of adjunctive glutamatergic therapies in treating symptoms that have not responded to antipsychotic medication (Javitt, 2004). The greater efficacy of clozapine compared with other antipsychotics, and its effectiveness in otherwise treatmentresistant patients, has been linked to its glutamatergic actions (Javitt et al, 2005). It has thus been suggested that patients who are still sympto matic following antipsychotic medication may exhibit persistent glutamatergic abnormalities, and may therefore benefit from treatments that act on the glutamate system (Stone et al, 2010).

Proton magnetic resonance imaging (1H-MRS) studies at field strengths of 3 Tesla or above in antipsychotic naive or minimally medicated first-episode psychosis patients have identified increases in the glutamate metabolite glutamine (Gln) or the Gln/glutamate ratio in the anterior cingulate cortex (Bustillo et al, 2010; Theberge et al, 2002), increases in Gln in the thalamus (Theberge et al, 2002), and increases in glutamate in the associative striatum (de la FuenteSandoval et al, 2011). This suggests that brain glutamatergic activity is increased in the early stages of the disorder, when patients are usually experiencing acute psychotic symptoms. 1H-MRS studies of the glutamate system in medicated 
patients with chronic schizophrenia have produced less consistent findings (Jessen et al, 2011; Reid et al, 2011; Shirayama et al, 2010; Tayoshi et al, 2009; Theberge et al, 2003; Wood et al, 2007). A recent meta-analysis indicated that although frontal glutamatergic activity may be elevated in untreated patients in the early phase of schizophrenia, reductions in frontal glutamate and Gln are evident in older patients at later stages of the disorder (Marsman et al, 2011). This apparent difference between the early and later stages of the disorder is not simply an effect of cumulative antipsychotic exposure, as prolonged treatment (for up to 80 months) is not associated with a change in glutamate metabolites in the anterior cingulate cortex (Aoyama et al, 2011; Bustillo et al, 2010; Theberge et al, 2007).

Some of the differences in the findings in studies of glutamate in schizophrenia may be related to differences in the symptom profiles of the patients studied, as glutamate metabolite levels may vary with the level of negative symptoms (Reid et al, 2011), cognitive deficits (Bustillo et al, 2011; Shirayama et al, 2010), and global functioning (Aoyama et al, 2011; Tibbo et al, 2004; Van Elst et al, 2005). In the present study, we tested the hypothesis that glutamate metabolite levels in the anterior cingulate cortex and thalamus would be higher in first-episode psychosis patients who were symptomatic compared with those with minimal symptoms following treatment.

\section{PATIENTS AND METHODS}

The study was approved by the Joint South London and Maudsley and the Institute of Psychiatry Research Ethics Committee. All subjects gave written informed consent to participate. Thirty-two patients who had presented with a first episode of schizophrenia (as defined by DSM-IV criteria), and who had completed at least one course of treatment with antipsychotic medication were recruited from the South London and Maudsley NHS Foundation Trust. Only patients who had presented within the past 2 years, and who had experienced a single episode of psychosis were included. The mean $\pm S D$ time since first presentation was 10.6 \pm 7.1 months. Exclusion criteria included a history of head injury, concomitant medical or neurological illness, drug or alcohol dependence (except nicotine), presence of metallic implants contraindicated at 3 Tesla, and pregnancy. Current and previous use of nicotine, cannabis, alcohol and antipsychotic medication, and family history of psychiatric illness were determined through self-report data. Symptoms at the time of imaging were assessed using the Positive and Negative Syndrome Scale (PANSS; Kay et al, 1987) and global functioning was assessed using the global assessment of functioning scale (Hall, 1995). Symptom severity scores were used to allocate patients to group (symptomatic remission $v s$ no remission) according to established criteria (Andreasen et al, 2005). Symptomatic remission was defined as having scores of mild or less on the all eight of the following PANSS positive $(\mathrm{P})$, negative $(\mathrm{N})$, or general $(\mathrm{G})$ syndrome scale items: Delusions (P1); Conceptual Disorganization (P2); Hallucinatory behavior (P3); Blunted affect (N1); Social withdrawal (N4); Lack of spontaneity (N6); Mannerisms/posturing (G5), and Unusual thought content (G9) (Andreasen et al,
2005). No remission was defined as having scores of moderate or more on the same items.

All scans were acquired on a General Electric (Milwaukee, Wisconsin) 3 Tesla HDx magnetic resonance system as detailed previously (Stone et al, 2009). An initial localizer scan was followed by acquisition of structural images, including an axial 2D T2-weighted fast spin echo scan and an axial fast fluid-attenuated inversion recovery scan. $1 \mathrm{H}$-MRS voxels were prescribed in the anterior cingulate cortex and left thalamus. The anterior cingulate cortex voxel was prescribed from the midline sagittal localizer, with the centre of the $20 \times 20 \times 20 \mathrm{~mm}$ voxel placed $13 \mathrm{~mm}$ above the genu of corpus callosum perpendicular to the AC-PC line (Figure 1a). A second (15 (right-left) $\times 20 \times 20 \mathrm{~mm}$ ) voxel was placed in centre of the left thalamus to include the maximum amount of grey matter (Figure 1b). ${ }^{1} \mathrm{H}$-MRS spectra (PRESS - Point RESolved Spectroscopy; TE = $30 \mathrm{msec}$; $\mathrm{T} r=3000 \mathrm{msec}$; 96 averages) were acquired using the standard GE PROBE (proton brain examination) sequence, which uses a standardized chemically selective suppression water suppression routine. Shimming was optimized, with auto-prescan performed twice before each scan.

\section{Data Analysis}

Spectra were analyzed using LCModel version 6.1-4 F (Provencher, 1993), using a standard basis set of 16 metabolites (L-alanine, aspartate, creatine, phosphocreatine, GABA, glucose, Gln, glutamate, glycerophosphocholine, glycine, myo-inositol, L-lactate, $\mathrm{N}$-acetylaspartate, $\mathrm{N}$-acetylaspartylglutamate, phosphocholine, and taurine), acquired with the same field strength ( 3 Tesla), localization sequence (PRESS), and echo time (30 msec). Model metabolites and concentrations used in the basis set are fully detailed in the LCModel manual (http://s-provencher-.com/pages/lcmmanual.shtml). Poorly fitted metabolite peaks (Cramer-Rao minimum variance bounds of $>20 \%$ as reported by LCModel) were excluded from further analysis. As information on voxel cerebrospinal fluid content was not available for correction of water-scaled metabolite concentration estimates, the ratio of the reported metabolite concentration to that of the creatine (Cr) plus phosphocreatine resonance was used for analysis.

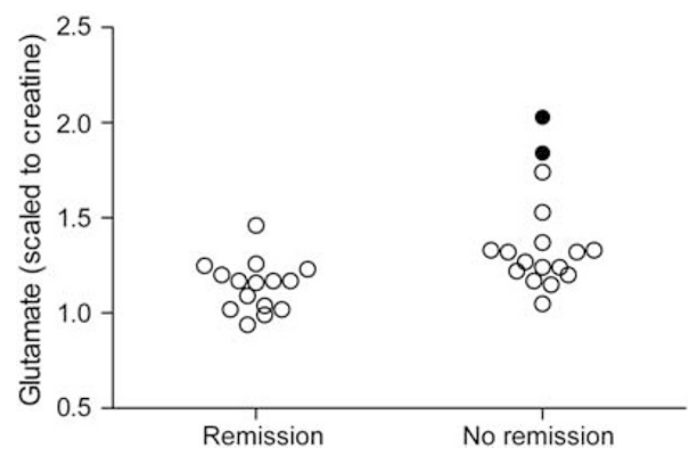

Figure I Level of glutamate (scaled to creatine) in the anterior cingulate cortex. Glutamate levels were significantly higher in non-remitted $(n=17)$ than remitted $(n=15)$ schizophrenia patients $(T(30)=3.02 ; P=0.005)$. This difference remained significant $\mathrm{T}(28)=2.79 ; P=0.009)$ after removal of the two outlying values as identified by Cook's $D$ and indicated by the filled symbols. 
Statistical analysis was performed in SPSS version 15.0 (Chicago, Illinois). Group differences in demographic and clinical variables, spectral quality and glutamate/Cr ratios were explored using Fisher's exact test or independentsamples $t$-tests as appropriate. Levene's test was used to check for equality of variance across groups. Statistical significance was defined as $P<0.05$. Relationships between glutamate/Cr ratio and symptom scores were explored using Pearson's product moment correlation, correcting for the number of correlations performed (threshold $P=0.05 / 4$ comparisons $=0.013$ ). Potential influences of age, medication status, or outlying values (as indicated by Cook's D) on significant findings were subsequently determined using partial correlation or exclusion of specific cases.

\section{RESULTS}

\section{Clinical and Demographic Characteristics}

Clinical and demographic variables are presented in Table 1. Fifteen patients met the criteria for symptomatic remission (mean \pm SD age $=30 \pm 6$ years, 11 male, 14 right handed), while seventeen were still symptomatic ('no remission'; mean $\pm S D$ age $=26 \pm 7$ years, 13 male, 17 right handed). There were no significant group differences in age, gender, handedness, or current tobacco, alcohol or cannabis use, or history of amphetamine, cocaine, ecstasy or ketamine use. In the symptomatic remission group, one patient was receiving amisulpride, one aripiprazole, five olanzapine, three risperidone, one quetiapine, and four were no longer receiving any antipsychotic medication. In the symptomatic non-remission group, one patient was receiving amisulpride, seven aripiprazole, three olanzapine, three risperidone, two quetiapine, and one was no longer taking any antipsychotic medication. There was no significant difference in the type of antipsychotic medication between groups. As expected, symptom severity on all PANSS subscales and the level of global functioning were significantly worse in the no remission compared with the remission group (Table 1).

\section{Quality of 1H-MRS Spectra}

Representative spectra for the anterior cingulate cortex and left thalamus voxels are provided in Supplemental Figure S1 available online. Serial water-scaled phantom data did not provide any evidence of step changes or scanner drift over the course of the study. Spectral quality was assessed as the NAA signal to noise ratios $(\mathrm{S} / \mathrm{N}$, defined as the ratio of the peak height at $2.01 \mathrm{ppm}$ minus baseline to twice the root mean square of the residuals of the fit, as reported by LCModel) and linewidths as reported by LCModel. Spectra were of good quality in the anterior cingulate cortex and thalamus and there were no significant group differences in spectral quality in either region (anterior cingulate cortex: $\mathrm{S} / \mathrm{N}$ mean $\pm \mathrm{SD}$ remission $=22.3 \pm 4.2$; no remission $20.0 \pm$ $5.0 ; \mathrm{t} 30=1.42 ; \quad P=0.17 ;$ linewidth $\pm \mathrm{SD}$ remission $=$ $0.039 \pm 0.007 \mathrm{ppm} ;$ no remission $0.038 \pm 0.005 \mathrm{ppm}$; $P=0.49$; or in the left thalamus (S/N mean $\pm \mathrm{SD}$ remission $=19.1 \pm 2.7$; no remission $18.5 \pm 3.5 ; \mathrm{t} 29=0.58$; $P=0.56$; linewidth $\pm \mathrm{SD}$ remission $=0.056 \pm 0.018 \mathrm{ppm}$; no remission $0.047 \pm 0.007 \mathrm{ppm} ; \mathrm{t} 29=1.78 ; P=0.09$ ). The mean \pm SD\% CRLB for each metabolite in each voxel for remitted and non-remitted patients are presented in Supplemental Table 1 available online. There were no significant group differences in metabolite \%CRLBs ( $P$ values range from 0.07 to 0.95 ).

Table I Demographic and Clinical Characteristics

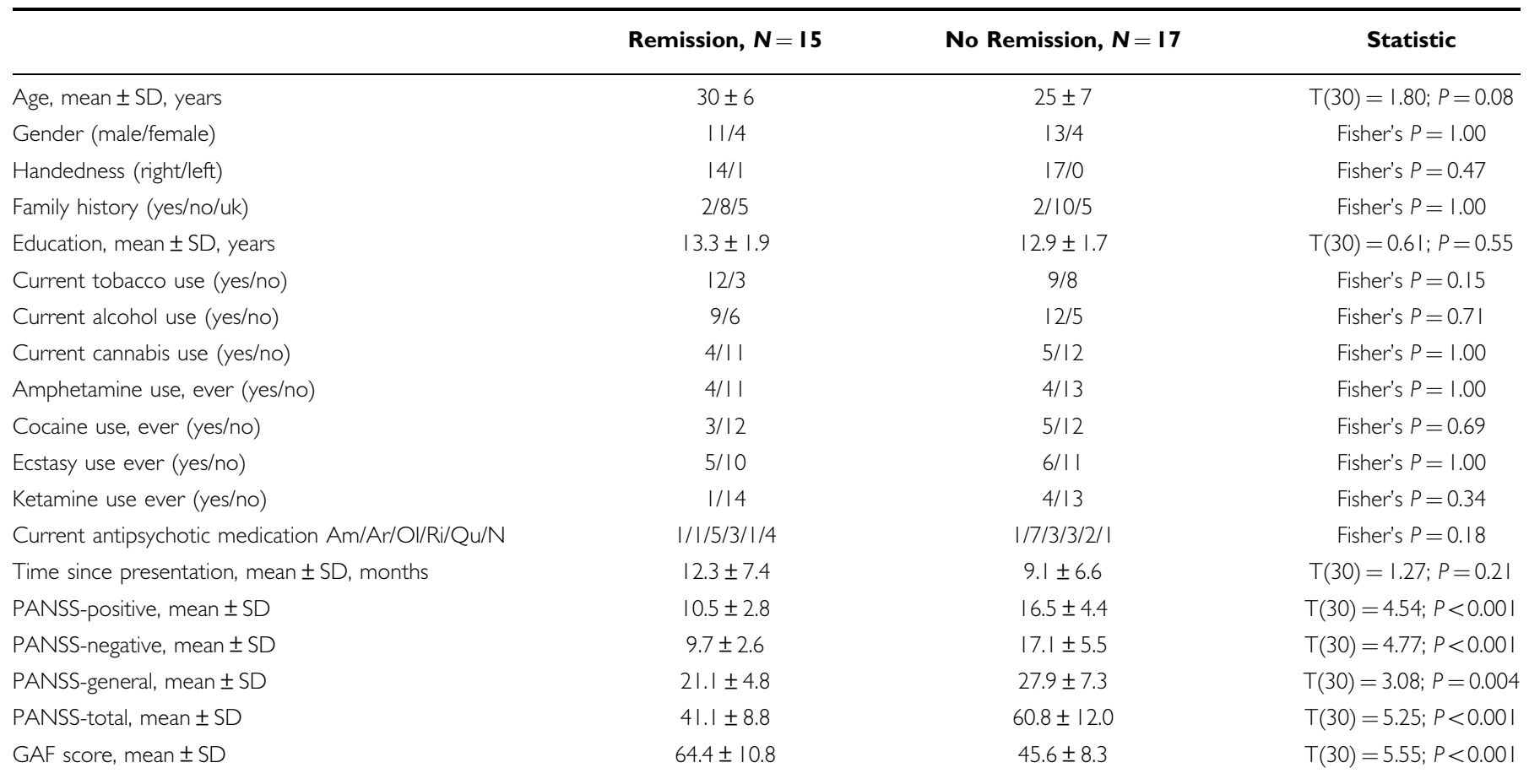

Abbreviations: Am, amisulpride; Ar, aripiprazole; N, none; Ol, olanzapine; Qu, quetiapine; Ri, risperidone; uk, unknown. 


\section{Metabolite Levels in Remitted vs Non Remitted Patients}

The non-remitted patients had significantly higher levels of glutamate/Cr in the anterior cingulate cortex than those in remission (Figure 1; $\mathrm{T}(30)=3.02 ; P=0.005$ ). There was a tendency towards higher variance in glutamate/Cr estimates in the no remission compared with the remission group (Levene's test: $\mathrm{F}=3.54 ; P=0.07$ ), and the group difference in glutamate/Cr remained significant accounting for inhomogeneity of variance $(\mathrm{T}(24.3)=3.14 ; P=0.004)$ and after removal of two outlying values (as identified by Cook's $\mathrm{D}$ and shown in Figure 1; $\mathrm{T}(28)=2.79 ; P=0.009$ ). The higher levels of anterior cingulate glutamate/Cr in nonremitted patients compared with remitted patients also remained significant after exclusion of the five patients no longer taking antipsychotics $(\mathrm{T}(25)=2.36 ; P=0.026)$, and when analyzed as water-scaled glutamate levels $(\mathrm{T}(30)=$ 2.23; $P=0.03$ ). Across all patients, there was a significant decline in anterior cingulate cortex glutamate/Cr with increasing age $(r=-0.425 ; P=0.015)$. However, the group difference in glutamate/Cr remained significant after controlling for age effects $(\mathrm{F}(1,31)=5.774 ; P=0.023)$. There were no significant effects of current tobacco (mean $\pm S D, n$ : non-smokers $1.29 \pm 0.27,11$; smokers $1.25 \pm 0.23,21$; $\mathrm{T}(30)=0.45 ; P=0.67$ ), current alcohol use (mean $\pm \mathrm{SD}, n$ : non-alcohol drinkers $1.24 \pm 0.24$, 11; alcohol drinkers $1.28 \pm 0.24,21 ; \mathrm{T}(30)=0.44 ; P=0.66$ ), current cannabis use (mean \pm s.d., $n$ : non-cannabis users $1.27 \pm 0.26$, 9; cannabis users $1.26 \pm 0.24,23 ; \mathrm{T}(30)=0.11 ; P=0.91$ ), or history of ever using other recreational drugs (amphetamine: $\mathrm{T}(30)=0.87 ; P=0.39$; cocaine: $\mathrm{T}(30)=0.51 ; P=0.61$; ecstasy: $\mathrm{T}(30)=0.39 ; P=0.70$; ketamine: $\mathrm{T}(30)=0.33 ; P=0.74$ ) on anterior cingulate glutamate/Cr levels, and no significant relationship between time since presentation and anterior cingulate glutamate/Cr levels $(n=32 ; r=-0.05 ; P=0.77)$. Although there was no significant group difference in glutamate plus $\mathrm{Gln}(\mathrm{Glx}) / \mathrm{Cr}(\mathrm{T}(30)=1.24 ; P=0.223)$, levels of $\mathrm{Glx} / \mathrm{Cr}$ were higher in non-remitted than remitted patients following the removal of three outlying values as identified with Cook's D (Figure 2, $\mathrm{T}(27)=2.20 ; P=0.036$ ). There were no significant group differences in the other metabolites quantifiable in the anterior cingulate cortex

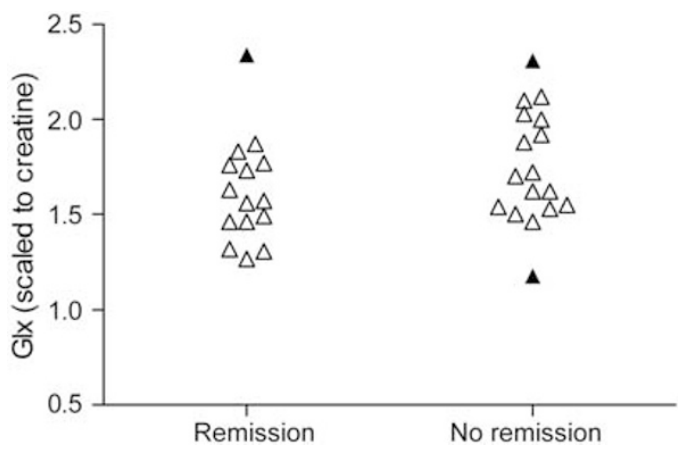

Figure 2 Level of Glx (glutamate plus glutamine, scaled to creatine) in the anterior cingulate cortex. There was no significant group difference in Glx including all cases $(T(30)=1.24 ; P=0.223)$, but levels of $\mathrm{Gl} / \mathrm{C} / \mathrm{Cr}$ were significantly higher in non-remitted than remitted patients $(T(27)=2.20$; $P=0.036$ ) after removal of three outlying values as identified with Cook's $\mathrm{D}$ and indicated by the filled symbols. spectra, including $\mathrm{Cr}$ (Table 2), and no significant group differences in any of the metabolites quantifiable in the left thalamus (Table 2).

\section{Relationships with Symptoms}

Across the entire sample, higher levels of glutamate/Cr in the anterior cingulate cortex were associated with a greater severity of negative symptoms (Figure $3 \mathrm{a} ; r=0.42$; $P=0.017$ ) and a lower level of global functioning (Figure $3 \mathrm{~b}$; $r=-0.47 ; P=0.007)$, but not severity of positive $(r=0.232$; $P=0.202)$ or general $(r=0.071 ; P=0.698)$ symptoms. The relationship between anterior cingulate cortex glutamate/ $\mathrm{Cr}$ and global functioning survived correction for multiple comparisons (threshold $P=0.05 / 4$ comparisons = $0.013)$. The relationships between anterior cingulate cortex glutamate/Cr and negative symptoms or global functioning remained significant after exclusion of the five patients no longer taking antipsychotic medication (PANSS negative: $r=0.401 ; P=0.038$; global functioning: $r=-0.436 ; P=0.023)$, and after controlling for age (PANSS negative: $r=0.465 ; P=0.008$; global functioning: $r=-0.447 ; P=0.012$ ). Significant relationships between anterior cingulate $\mathrm{Glx} / \mathrm{Cr}$ and PANSS negative symptom severity $(r=0.384 ; P=0.040)$ and global functioning ( $r=-0.404 ; P=0.030)$ were also evident after exclusion of three outlying values as identified with Cook's D. No significant relationships between symptoms and either glutamate or Glx levels in the left thalamus were detected.

Table 2 IH-MRS Metabolite Levels (mean \pm SD) in the Anterior Cingulate Cortex and Left Thalamus in First Episode Psychosis Patients Who were or were not in Symptomatic Remission Following Antipsychotic Treatment

\begin{tabular}{|c|c|c|c|}
\hline & $\begin{array}{c}\text { Remission } \\
\text { Mean } \pm \text { SD, n }\end{array}$ & $\begin{array}{l}\text { No Remission } \\
\text { Mean } \pm \text { SD, n }\end{array}$ & Statistic \\
\hline \multicolumn{4}{|l|}{$\begin{array}{l}\text { Anterior cingulate } \\
\text { cortex }\end{array}$} \\
\hline Creatine & $6.28 \pm 0.43,15$ & $6.05 \pm 1.00,17$ & $\mathrm{~T}(30)=0.8 \mathrm{I} ; P=0.42$ \\
\hline Glutamate/Cr & $1.14 \pm 0.13,15$ & $1.37 \pm 0.26,17$ & $\mathrm{~T}(30)=3.02 ; P=0.005$ \\
\hline $\mathrm{Gl} / \mathrm{Cr}$ & $1.62 \pm 0.28,15$ & $1.75 \pm 0.30,17$ & $\mathrm{~T}(30)=1.25 ; P=0.22$ \\
\hline $\mathrm{NAA} / \mathrm{Cr}$ & $1.25 \pm 0.12,15$ & $1.28 \pm 0.11,17$ & $\mathrm{~T}(30)=0.72 ; P=0.48$ \\
\hline Choline/Cr & $0.26 \pm 0.03,15$ & $0.27 \pm 0.04,17$ & $\mathrm{~T}(30)=0.79 ; P=0.44$ \\
\hline $\mathrm{ml} / \mathrm{Cr}$ & $0.87 \pm 0.13,15$ & $0.88 \pm 0.12,17$ & $T(30)=0.18 ; P=0.86$ \\
\hline \multicolumn{4}{|l|}{ Left thalamus } \\
\hline Creatine & $6.63 \pm 0.60,14$ & $6.92 \pm 0.74,17$ & $\mathrm{~T}(29)=1.19 ; P=0.24$ \\
\hline Glutamate/Cr & $0.92 \pm 0.16,13$ & $1.02 \pm 0.23,16$ & $\mathrm{~T}(27)=1.40 ; P=0.17$ \\
\hline $\mathrm{Gl} / \mathrm{Cr}$ & $1.16 \pm 0.28,13$ & $1.28 \pm 0.29,15$ & $T(26)=1.10 ; P=0.28$ \\
\hline $\mathrm{NAA} / \mathrm{Cr}$ & $1.70 \pm 0.20,14$ & $1.76 \pm 0.25,17$ & $\mathrm{~T}(29)=0.82 ; P=0.42$ \\
\hline Choline/Cr & $0.28 \pm 0.04,14$ & $0.28 \pm 0.02,17$ & $\mathrm{~T}(29)=0.50 ; P=0.62$ \\
\hline $\mathrm{ml} / \mathrm{Cr}$ & $0.63 \pm 0.14,14$ & $0.58 \pm 0.11,17$ & $\mathrm{~T}(29)=1.06 ; P=0.30$ \\
\hline
\end{tabular}

Abbreviations: Choline, total choline plus phosphocholine. $\mathrm{Cr}$, creatine plus phosphocreatine; Glx, glutamate plus glutamine; NAA, total N-acetyl aspartate plus $\mathrm{N}$-acetyl aspartyl glutamic acid. 

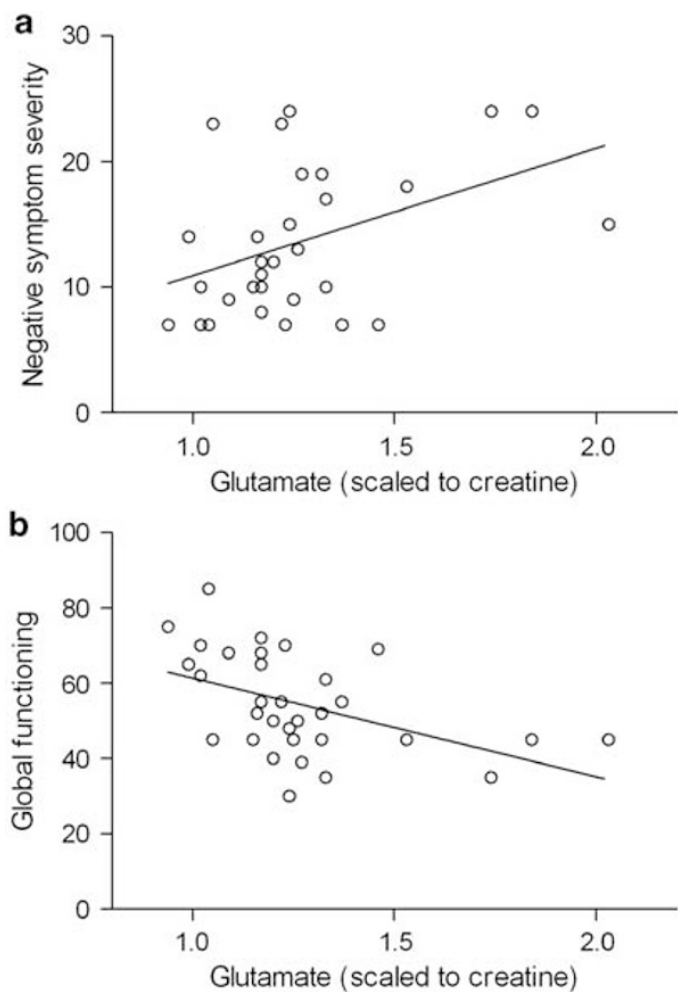

Figure 3 Higher levels of glutamate (scaled to creatine) in the anterior cingulate cortex were associated with (a) greater severity of negative symptoms (left; $r=0.42 ; P=0.017$ ), and with (b) lower levels of global functioning (right; $r=-0.47 ; P=0.007$ ).

\section{DISCUSSION}

To our knowledge, this is the first evidence that anterior cingulate glutamate levels differ between patients with schizophrenia in remission following antipsychotic treatment and patients who are still symptomatic. Higher levels of glutamate and Glx in the anterior cingulate cortex in symptomatic compared with remitted patients are consistent with reports of elevated anterior cingulate glutamate turnover in non-medicated or minimally treated patients, who generally have high levels of psychotic symptoms (Bustillo et al, 2010; Theberge et al, 2002). It is also in line with evidence that frontal glutamate is reduced in chronic patients with a long history of antipsychotic treatment and who are generally less symptomatic (Marsman et al, 2011; Tayoshi et al, 2009; Theberge et al, 2003). Our finding is broadly consistent with the numerous reports of abnormalities in the anterior cingulate cortex in early psychosis. For example, structural abnormalities in this area are present at onset of psychosis and may be predictive of conversion to psychosis in those at high risk of developing the disorder (Borgwardt et al, 2007; Dazzan et al, 2011; Fornito et al, 2008; Pantelis et al, 2003; Rothlisberger et al, 2012; Fusar-Poli et al, 2011). Moreover, functional magnetic resonance imaging studies show that antipsychotic treatment may be particularly effective in normalizing activity in the anterior cingulate cortex ((Snitz et al, 2005) and see (Karch et al, 2012) for review). Our finding may suggest that this is linked to glutamatergic function.
We also found that higher levels of anterior cingulate glutamate or Glx were associated with both an increased severity of negative symptoms and a lower level of global functioning. A relationship between glutamate levels and global functioning is in accordance with similar findings in patients with chronic schizophrenia (Van Elst et al, 2005), while the association with negative symptoms is consistent with an inverse relationship between anterior cingulate glutamate levels and sensation seeking (Gallinat et al, 2007). However, negative symptom severity has also been linked to reduced anterior cingulate glutamate levels in stable, medicated schizophrenia patients (Reid et al, 2011). The reason for the difference in the direction of the correlation in this and the present study is unclear, but may reflect differences in the part of the anterior cingulate cortex from which spectra were acquired, or in the characteristics of the respective patient samples.

No differences in left thalamic metabolite concentrations were detected between remitted and non-remitted patients. Previous studies suggest that increased thalamic Gln levels in never-treated first-episode patients return to normal after 30 , but not 10 months of antipsychotic treatment (Theberge et al, 2007), and that thalamic Glx levels decrease over 80 months of treatment (Aoyama et al, 2011). Given that in the present study the average length of time since presentation was 10 months, and both groups had similar treatment histories, the absence of group differences is unlikely to be related to an effect of antipsychotic treatment.

One limitation of the present study is that in the majority of spectra, we were unable to reliably quantify Gln concentrations. At field strengths of 3 Tesla or below, glutamate and Gln are difficult to separate due to overlapping resonances (Hancu, 2009). It is therefore possible that in some cases, glutamate values may have been contaminated by Gln. In this study, metabolite concentrations were reported in ratio to Cr. Compared with absolute quantitation, this approach is associated with high levels of accuracy, resilient to variation in signal-to-noise ratio (Kanowski et al, 2004). The limitation of this method is that it assumes there is no group difference in $\mathrm{Cr}$ concentration. However, our findings were significant both when assessed as the estimate of water-scaled glutamate concentration, and as the ratio of estimated glutamate concentration to $\mathrm{Cr}$, and furthermore, there was no group difference in estimates of absolute $\mathrm{Cr}$ concentration. A further limitation is that glutamate levels are higher in grey than white matter (Pan et al, 1996; Pouwels and Frahm, 1998) and, as we did not assess voxel tissue content, the influence of individual differences in voxel percentage grey and white matter on glutamate levels cannot be determined.

Although there was no significant difference in medication type between patients who were or were not in remission, the sample comprised patients on a variety of different antipsychotics, and medication adherence was assessed through self-report. The absence of precise information on duration of antipsychotic treatment is a weakness, although at the recruitment site, all first-episode patients are prescribed antipsychotics, ideally for the first 2 years, and the duration of treatment is correlated with the time since first presentation. Antipsychotics may differentially impact on regional glutamatergic function (McLoughlin et al, 2009), and their potentially confounding effects could be better 
assessed in a prospective design involving a standardized treatment protocol or monitoring of prescribing and adherence from presentation. As we did not exclude subjects on the basis of recreational drug use, or perform urine drugs screens at the time of imaging, we cannot exclude the possibility that drug use may have impacted on our findings. However, there were no significant group differences in selfreported recreational drug use or differences in anterior cingulate glutamate/Cr levels between subjects who did or did not report use of each drug. Finally, as our study was designed to compare patients who were symptomatic vs remitted following treatment, we did not include a healthy control group, therefore we could not assess whether the metabolite levels in the patients differed from those in healthy volunteers.

One interpretation of our findings is that within our sample of patients with schizophrenia, pre-existing differences in anterior cingulate glutamate levels predicted the subsequent response to antipsychotic treatment. However, we cannot exclude the possibility that the entire sample had elevated glutamate levels before treatment and that there was a longitudinal reduction in the patients who reached criteria for symptomatic remission, or that less severe symptoms were also associated with lower anterior cingulate glutamate levels before treatment. Prospective studies are required to address these issues. Nonetheless, our findings suggest that presence of residual symptoms in schizophrenia may be linked to glutamate dysfunction. Patients not in remission following initial antipsychotic treatment may therefore benefit from treatment with antipsychotics that have glutamatergic as well as dopaminergic actions (eg, clozapine), or other drugs that act on the glutamate system.

\section{ACKNOWLEDGEMENTS}

This work was supported by the NIHR Biomedical Research Centre for Mental Health at the South London and Maudsley NHS Foundation Trust and Institute of Psychiatry, King's College London. We thank the study participants and the radiography team at the Centre for Neuroimaging Sciences, Institute of Psychiatry, King's College London.

\section{DISCLOSURE}

GJB received honoraria for teaching from General Electric during the course of this work. In the past 3 years, JS has received a non-restricted academic fellowship from Glaxo SmithKline, and honoraria from Roche, AstraZeneca, Behrenberg Bank, and Pfizer. The authors declare no conflict of interest.

\section{REFERENCES}

Andreasen NC, Carpenter Jr WT, Kane JM, Lasser RA, Marder SR, Weinberger DR (2005). Remission in schizophrenia: proposed criteria and rationale for consensus. Am J Psychiatry 162: 441-449.

Aoyama N, Theberge J, Drost DJ, Manchanda R, Northcott S, Neufeld RW et al. (2011). Grey matter and social functioning correlates of glutamatergic metabolite loss in schizophrenia. $\mathrm{BrJ}$ Psychiatry 198: 448-456.
Borgwardt SJ, McGuire PK, Aston J, Berger G, Dazzan P, Gschwandtner U et al. (2007). Structural brain abnormalities in individuals with an at-risk mental state who later develop psychosis. Br J Psychiatry Suppl 51: s69-s75.

Bustillo JR, Chen H, Gasparovic C, Mullins P, Caprihan A, Qualls C et al. (2011). Glutamate as a marker of cognitive function in schizophrenia: a proton spectroscopic imaging study at 4 Tesla. Biol Psychiatry 69: 19-27.

Bustillo JR, Rowland LM, Mullins P, Jung R, Chen H, Qualls C et al. (2010). (1)H-MRS at 4 Tesla in minimally treated early schizophrenia. Mol Psychiatry 15: 629-636.

Dazzan P, Soulsby B, Mechelli A, Wood SJ, Velakoulis D, Phillips LJ et al. (2011). Volumetric abnormalities predating the onset of schizophrenia and affective psychoses: an MRI study in subjects at ultrahigh risk of psychosis. Schizophr Bull; e-pub ahead of print 25 April 2011.

de la Fuente-Sandoval C, Leon-Ortiz P, Favila R, Stephano S, Mamo D, Ramirez-Bermudez J et al. (2011). Higher levels of glutamate in the associative-striatum of subjects with prodromal symptoms of schizophrenia and patients with first-episode psychosis. Neuropsychopharmacology 36: 1781-1791.

Egerton A, Fusar-Poli P, Stone JM (2012). Glutamate and psychosis risk. Curr Pharm Des 18: 466-478.

Fornito A, Yung AR, Wood SJ, Phillips LJ, Nelson B, Cotton S et al. (2008). Anatomic abnormalities of the anterior cingulate cortex before psychosis onset: an MRI study of ultra-high-risk individuals. Biol Psychiatry 64: 758-765.

Fusar-Poli P, Radua J, McGuire P, Borgwardt S (2011). Neuroanatomical maps of psychosis onset: voxel-wise meta-analysis of antipsychotic-naive VBM studies. Schizophr Bull; e-pub ahead of print 10 November 2011.

Gallinat J, Kunz D, Lang UE, Neu P, Kassim N, Kienast T et al. (2007). Association between cerebral glutamate and human behaviour: the sensation seeking personality trait. Neuroimage 34: 671-678.

Hall RC (1995). Global assessment of functioning. A modified scale. Psychosomatics 36: 267-275.

Hancu I (2009). Optimized glutamate detection at 3T. J Magn Reson Imaging 30: 1155-1162.

Javitt DC (2004). Glutamate as a therapeutic target in psychiatric disorders. Mol Psychiatry 9: 984-997.

Javitt DC, Duncan L, Balla A, Sershen H (2005). Inhibition of system A-mediated glycine transport in cortical synaptosomes by therapeutic concentrations of clozapine: implications for mechanisms of action. Mol Psychiatry 10: 275-287.

Jessen F, Fingerhut N, Sprinkart AM, Kuhn KU, Petrovsky N, Maier W et al. (2011). N-acetylaspartylglutamate (NAAG) and $\mathrm{N}$-acetylaspartate (NAA) in patients with schizophrenia. Schizophr Bull; e-pub ahead of print 12 September 2011.

Kanowski M, Kaufmann J, Braun J, Bernarding J, Tempelmann C (2004). Quantitation of simulated short echo time $1 \mathrm{H}$ human brain spectra by LCModel and AMARES. Magn Reson Med 51: 904-912.

Kapur S, Zipursky R, Jones C, Remington G, Houle S (2000). Relationship between dopamine $\mathrm{D}(2)$ occupancy, clinical response, and side effects: a double-blind PET study of first-episode schizophrenia. Am J Psychiatry 157: 514-520.

Karch S, Pogarell O, Mulert C (2012). Functional magnetic resonance imaging and treatment strategies in schizophrenia. Curr Pharm Biotechnol 13: 1622-1629.

Kay SR, Fiszbein A, Opler LA (1987). The positive and negative syndrome scale (PANSS) for schizophrenia. Schizophr Bull 13: 261-276.

Marsman A, van den Heuvel MP, Klomp DW, Kahn RS, Luijten PR, Hulshoff Pol HE (2011). Glutamate in schizophrenia: a focused review and meta-analysis of $1 \mathrm{H}-\mathrm{MRS}$ studies. Schizophr Bull; e-pub ahead of print 11 July 2011.

McLoughlin GA, Ma D, Tsang TM, Jones DN, Cilia J, Hill MD et al. (2009). Analyzing the effects of psychotropic drugs on metabolite profiles in rat brain using $1 \mathrm{H}$ NMR spectroscopy. J Proteome Res 8: 1943-1952. 
Pan JW, Mason GF, Pohost GM, Hetherington HP (1996). Spectroscopic imaging of human brain glutamate by watersuppressed J-refocused coherence transfer at 4.1 T. Magn Reson Med 36: 7-12.

Pantelis C, Velakoulis D, McGorry PD, Wood SJ, Suckling J, Phillips LJ et al. (2003). Neuroanatomical abnormalities before and after onset of psychosis: a cross-sectional and longitudinal MRI comparison. Lancet 361: 281-288.

Pilowsky LS, Costa DC, Ell PJ, Murray RM, Verhoeff NP, Kerwin RW (1993). Antipsychotic medication, D2 dopamine receptor blockade and clinical response: a 123I IBZM SPET (single photon emission tomography) study. Psychol Med 23: 791-797.

Pouwels PJ, Frahm J (1998). Regional metabolite concentrations in human brain as determined by quantitative localized proton MRS. Magn Reson Med 39: 53-60.

Provencher SW (1993). Estimation of metabolite concentrations from localized in vivo proton NMR spectra. Magn Reson Med 30: 672-679.

Reid MA, Stoeckel LE, White DM, Avsar KB, Bolding MS, Akella NS et al. (2011). Assessments of function and biochemistry of the anterior cingulate cortex in schizophrenia. Biol Psychiatry 68: 625-633.

Rothlisberger M, Riecher-Rossler A, Aston J, Fusar-Poli P, Radu EW, Borgwardt S (2012). Cingulate volume abnormalities in emerging psychosis. Curr Pharm Des 18: 495-504.

Shirayama Y, Obata T, Matsuzawa D, Nonaka H, Kanazawa Y, Yoshitome E et al. (2010). Specific metabolites in the medial prefrontal cortex are associated with the neurocognitive deficits in schizophrenia: a preliminary study. Neuroimage 49: 2783-2790.

Snitz BE, MacDonald III A, Cohen JD, Cho RY, Becker T, Carter CS (2005). Lateral and medial hypofrontality in first-episode schizophrenia: functional activity in a medication-naive state and effects of short-term atypical antipsychotic treatment. $A m \mathrm{~J}$ Psychiatry 162: 2322-2329.

Stone JM, Day F, Tsagaraki H, Valli I, McLean MA, Lythgoe DJ et al. (2009). Glutamate dysfunction in people with prodromal symptoms of psychosis: relationship to gray matter volume. Biol Psychiatry 66: 533-539.

Stone JM, Morrison PD, Pilowsky LS (2007). Glutamate and dopamine dysregulation in schizophrenia-a synthesis and selective review. J Psychopharmacol 21: 440-452.

Stone JM, Raffin M, Morrison P, McGuire PK (2010). Review: the biological basis of antipsychotic response in schizophrenia. J Psychopharmacol 24: 953-964.

Tayoshi S, Sumitani S, Taniguchi K, Shibuya-Tayoshi S, Numata S, Iga J et al. (2009). Metabolite changes and gender differences in schizophrenia using 3-Tesla proton magnetic resonance spectroscopy (1H-MRS). Schizophr Res 108: 69-77.

Theberge J, Al-Semaan Y, Williamson PC, Menon RS, Neufeld RW, Rajakumar $\mathrm{N}$ et al. (2003). Glutamate and glutamine in the anterior cingulate and thalamus of medicated patients with chronic schizophrenia and healthy comparison subjects measured with 4.0-T proton MRS. Am J Psychiatry 160: 2231-2233.

Theberge J, Bartha R, Drost DJ, Menon RS, Malla A, Takhar J et al. (2002). Glutamate and glutamine measured with $4.0 \mathrm{~T}$ proton MRS in never-treated patients with schizophrenia and healthy volunteers. Am J Psychiatry 159: 1944-1946.

Theberge J, Williamson KE, Aoyama N, Drost DJ, Manchanda R, Malla AK et al. (2007). Longitudinal grey-matter and glutamatergic losses in first-episode schizophrenia. Br J Psychiatry 191: 325-334.

Tibbo P, Hanstock C, Valiakalayil A, Allen P (2004). 3-T proton MRS investigation of glutamate and glutamine in adolescents at high genetic risk for schizophrenia. Am J Psychiatry 161: 1116-1118.

Van Elst LT, Valerius G, Buchert M, Thiel T, Rusch N, Bubl E et al. (2005). Increased prefrontal and hippocampal glutamate concentration in schizophrenia: evidence from a magnetic resonance spectroscopy study. Biol Psychiatry 58: 724-730.

Wood SJ, Yucel M, Wellard RM, Harrison BJ, Clarke K, Fornito A et al. (2007). Evidence for neuronal dysfunction in the anterior cingulate of patients with schizophrenia: a proton magnetic resonance spectroscopy study at 3 T. Schizophr Res 94: 328-331.

Supplementary Information accompanies the paper on the Neuropsychopharmacology website (http://www.nature.com/npp) 